\title{
Optical modulation in a resonant tunneling relaxation oscillator
}

\author{
J. M. L. Figueiredo, ${ }^{\text {a) }}$ C. R. Stanley, A. R. Boyd, and C. N. Ironside \\ Department of Electronics and Electrical Engineering, University of Glasgow, Glasgow G12 8LT, United \\ Kingdom \\ S. G. McMeekin \\ Cardiff School of Engineering, University of Wales Cardiff, Cardiff NP2 1XH, United Kingdom
}

\author{
A. M. P. Leite \\ Centro de Física do Porto-ADFCUP, Universidade do Porto, Rua do Campo Alegre 687, 4169-007 Porto, \\ Portugal
}

(Received 16 July 1998; accepted for publication 22 December 1998)

\begin{abstract}
We report high-speed optical modulation in a resonant tunneling relaxation oscillator consisting of a resonant tunneling diode (RTD) integrated with a unipolar optical waveguide and incorporated in a package with a coplanar waveguide transmission line. When appropriately biased, the RTD can provide wide-bandwidth electrical gain. For wavelengths near the material band edge, small changes of the applied voltage give rise to large, high-speed electroabsorption modulation of the light. We have observed optical modulation at frequencies up to $14 \mathrm{GHz}$, associated with subharmonic injection locking of the RTD oscillation at the fundamental mode of the coplanar transmission line, as well as generation of $33 \mathrm{ps}$ optical pulses due to relaxation oscillation. (C) 1999 American
\end{abstract} Institute of Physics. [S0003-6951(99)01809-4]

Resonant tunneling diodes (RTDs) have been widely studied because of their potential application in high frequency signal generation, ${ }^{1}$ high speed signal processing at microwave frequencies, ${ }^{2}$ and in optoelectronics. ${ }^{3-6}$ Recent studies on resonant tunneling relaxation oscillators ${ }^{7,8}$ have shown generation of $30 \mathrm{ps}$ electrical pulses at a repetition rate of $1.1 \mathrm{GHz}$, and harmonic and subharmonic locking. In an optical waveguide containing a double-barrier RTD implemented in the AlGaAs material system, our group previously reported electroabsorption modulation at $900 \mathrm{MHz}$ with a modulation depth of $7 \mathrm{~dB} .{ }^{9}$ The modulation relies on a depletion region that is formed in the waveguide core; depending on the bias condition, a substantial part of the terminal voltage may be dropped across this region. From the current-voltage $(I-V)$ characteristic of the RTD optical waveguide modulator, it can be seen that small changes of bias voltage close to the peak-to-valley transition region can cause large changes in the electric field distribution across the depleted part of the waveguide core. The electric field shifts the absorption band edge to longer wavelengths via the Franz-Keldysh effect ${ }^{10}$ and therefore changes the transmission characteristics of the waveguide. Compared to the conventional $p n$ electroabsorption modulator, the advantage of the RTD modulator is that when dc biased close to the NDR region, the device behaves as an optical waveguide electroabsorption modulator integrated with a wide bandwidth electrical amplifier. This letter reports results of high speed light modulation in a resonant tunneling relaxation oscillator configuration, consisting of a RTD optical waveguide modulator integrated with a coplanar waveguide transmission line.

The RTD optical waveguide structure was grown by mo-

\footnotetext{
a) Also with the Centro de Física do Porto-ADFCUP, Universidade do Porto, Rua do Campo Alegre 687, 4169-007 PORTO, Portugal. Electronic mail: jlfiguei@fc.up.pt
}

lecular beam epitaxy in a Varian Gen II system, on a semiinsulating GaAs substrate [Fig. 1(a)]. It consists of two 1.4 $\mathrm{nm}$ thick AlAs barriers separated by a $7 \mathrm{~nm}$ wide GaAs quantum well, and two $500 \mathrm{~nm}$ thick moderately doped (Si: $2 \times 10^{16} \mathrm{~cm}^{-3}$ ) GaAs spacer layers each side of the double barrier, which are surrounded by heavily doped ( Si: $2 \times 10^{18} \mathrm{~cm}^{-3}$ ) AlGaAs cladding layers, allowing light confinement in the direction parallel to the double barrier plane. A $n+$ GaAs cap layer was provided for formation of $\mathrm{Au}-\mathrm{Ge}-\mathrm{Ni}$ ohmic contacts. The ridge waveguides $(2-6 \mu \mathrm{m}$ wide) and the large-area mesas each side of the ridges were

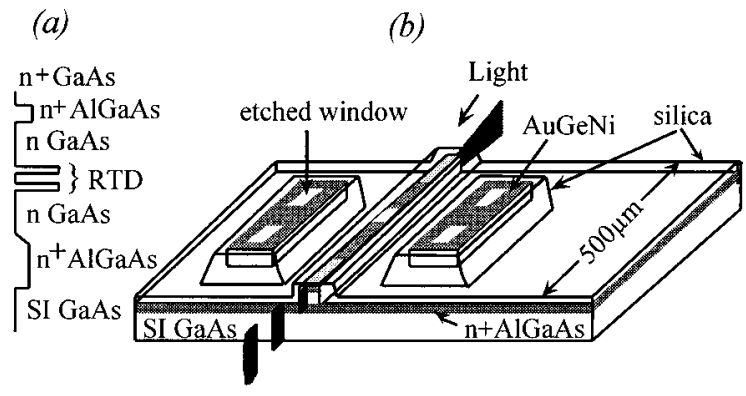

(c)

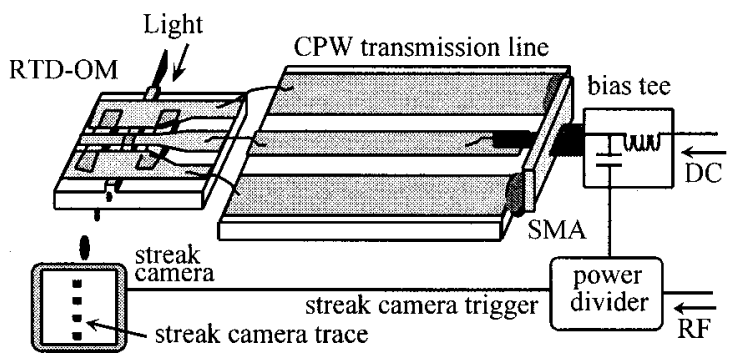

FIG. 1. (a) Schematic diagram of the wafer structure. (b) The RTD optical modulator (RTD-OM) configuration. (c) Diagram of a packaged device and measurement setup. 
fabricated by dry etching. Ohmic contacts $(100-400 \mu \mathrm{m}$ long) were deposited on top of the ridges and mesas. $\mathrm{A} \mathrm{SiO}_{2}$ layer was deposited, and access contact windows were etched on the ridge and the mesa electrodes [Fig. 1(b)], allowing contact to be made to high frequency bonding pads. After cleaving, the devices were die bonded on packages containing $50 \Omega$ coplanar waveguide (CPW) transmission lines of different lengths. The device pads were connected to the package CPW pads via gold wires; a SMA connector was then soldered to the package CPW line [Fig. 1(c)]. The dc and rf signals were applied through a $40 \mathrm{GHz}$ bandwidth bias tee. The dc $I-V$ characteristics of the packaged devices show typical RTD behavior, with the peak current density being approximately $13 \mathrm{kA} \mathrm{cm}^{-2}\left(800 \mu \mathrm{m}^{2}\right.$ active area) and a peak-to-valley current ratio around 1.5. Peak voltages were in the range $2.1-2.5 \mathrm{~V}$ and the valley voltage varied from 2.6 to $3.2 \mathrm{~V}$.

The optical characterization employed light from a Ti:sapphire laser, tunable in the wavelength region around the absorption edge of the GaAs waveguide (850-950 nm). Light was coupled into the waveguide by a microscope objective end-fire arrangement. To measure the change in the optical absorption spectrum induced by the peak-to-valley transition, a rf signal was injected to switch the RTD between the extremes of the NDR region, and a photodetector was used to measure the transmitted light. The band edge shift was found to be approximately $12 \mathrm{~nm}$. The high-speed optical response of the modulator was measured with a streak camera (Hamamatsu C5680) with a minimum time resolution of around $2 \mathrm{ps}$. A part of the injected rf signal power was required to trigger the streak camera. Figure 1(c) schematically presents the experimental setup.

Due to the highly nonlinear $I-V$ characteristic in the NDR region, the RTD is able to generate many high-order odd harmonics of the injected signal. ${ }^{8}$ We have studied optical modulation in a RTD optical waveguide relaxation oscillator due to injection locking of the RTD to the fundamental mode of a cavity formed by a transmission line. Optical modulation at $14 \mathrm{GHz}$ was observed in a packaged device with a $5 \mathrm{~mm}$ long transmission line, dc biased in the NDR region $(2.25 \mathrm{~V})$, when a rf signal of $0.4 \mathrm{~V}$ amplitude and frequency around $1 \mathrm{GHz}$ was injected. A packaged device with a $7 \mathrm{~mm}$ long transmission line, again dc biased in the NDR region $(2.4 \mathrm{~V})$ showed optical modulation at $8 \mathrm{GHz}$ for an injected signal of $0.6 \mathrm{~V}$ amplitude and frequency around 2 GHz. The streak camera traces for the 14 and $8 \mathrm{GHz}$ optical responses are presented in Fig. 2. In both cases, switching occurs between the extremes of the NDR region, producing sinusoidallike oscillating wave forms. At this stage the cavity comprised only the modulator CPW and the package CPW terminated with the SMA connector. The left side of the modulator CPW is unterminated (open circuit), as indicated in Fig. 1(c). The SMA connector limits the apparatus bandwidth to $18 \mathrm{GHz}$. By inserting coaxial transmission lines between the packaged device and the bias tee, we also observed modulation at the coaxial transmission lines fundamental mode frequency. Figure 3(a) shows a diagram of the equivalent electrical circuit. The modulation efficiency of the RTD optical modulator, characterized by the bandwidth-todrive-voltage ratio which is defined as the ratio of the opera- (a)

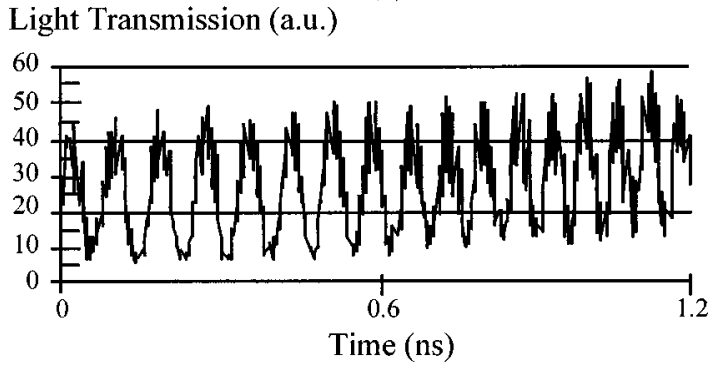

(b)

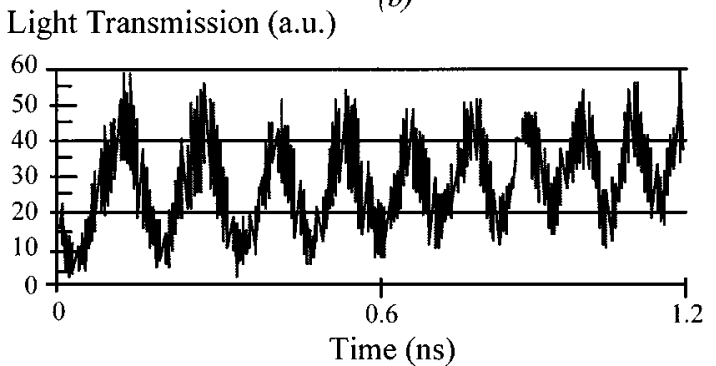

FIG. 2. Streak camera traces of the modulator optical response associated with locking of the RTD oscillation to the fundamental mode of a package with two different transmission lines. (a) Trace corresponding to $14 \mathrm{GHz}$ optical modulation for a packaged device with a CPW transmission line 5 $\mathrm{mm}$ long. (b) Trace of the $8 \mathrm{GHz}$ modulator optical response for a packaged device with a CPW transmission line $7 \mathrm{~mm}$ long.

tion bandwidth to the operating voltage for the $10 \mathrm{~dB}$ modulation depth, is higher than $33 \mathrm{GHz} / \mathrm{V}$.

When scanning the frequency of the injected signal with amplitude in the range $0.8-1.4 \mathrm{~V}$, it was found that the packaged RTD optical modulator connected through a coaxial line $30 \mathrm{~cm}$ long, produced optical pulses with a full width at half maximum (FWHM) of approximately $33 \mathrm{ps}$, at frequen-

(a)

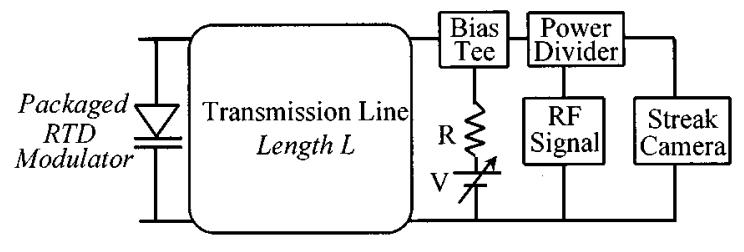

(b)

Light Transmission (a.u.)

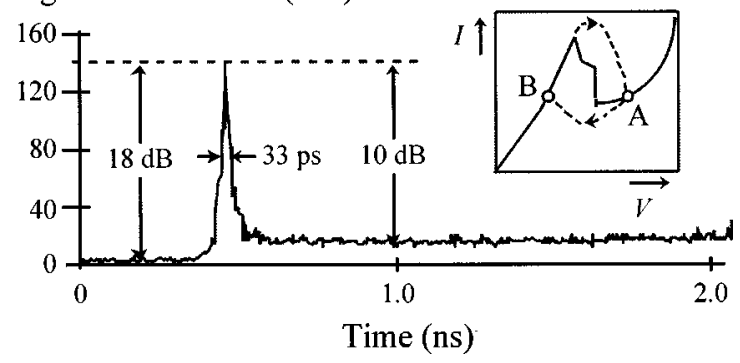

FIG. 3. (a) Schematic diagram of the electrical circuit. (b) Streak camera measurement of the 33 ps pulse optical response, showing modulation up to $18 \mathrm{~dB}$. 
cies also associated with the device operation as a relaxation oscillator. ${ }^{7}$ Figure 3(b) displays the streak camera trace of an optical pulse with 33 ps FWHM. According to Ref. 7, the pulses are caused by the discharge of the RTD capacitance when the RTD switches from the second positive differential resistance (PDR) region to the first PDR region. On a longer time scale other streak camera results indicate a pulse repetition rate around $166 \mathrm{MHz}$, further confirmed by analysis of the optical signal with a $2 \mathrm{GHz}$ bandwidth photodetector. The oscillation wave forms were square wavelike. For a 15 $\mathrm{cm}$ long coaxial line the pulse repetition rate was around 340 MHz. The trace in Fig. 3(b) shows a maximum modulation depth of $18 \mathrm{~dB}$, which suggests that the RTD now switches between two astable points well into the PDR regions. If we follow the explanation of Ref. 7 and the inset in Fig. 3(b), when starting at point A (the upper dwell zone of the relaxation oscillator) the collector is fully depleted and the electric field is high, giving low transmission. A return pulse forces the trajectory down below the valley, turning up a few ps later, toward point B (the lower dwell zone). During switching, the RTD capacitance first discharges, strongly decreasing the electric field across the depleted region, which gives rise to high transmission. The RTD capacitance starts to recharge causing the electric field to rise to the astable value associated with the first dwell zone. Transmission at point B is higher than at point $\mathrm{A}$, because the collector region is now not fully depleted. Switching from B to A depletes the entire collector region, recharging the RTD capacitance and making the transmission drop again. This drop is not evident in Fig. 3(b) because of the short time scale operation of the streak camera required to resolve the 33 ps pulse.

In conclusion, integrating a RTD with an optical waveguide is a relatively easy way of combining a wide band- width electrical amplifier with an electroabsorption modulator, opening up the possibility of a variety of modes of operation. A RTD optical waveguide modulator in a relaxation oscillator configuration has been demonstrated, providing optical modulation up to $14 \mathrm{GHz}$ when a $0.4 \mathrm{~V}$ amplitude rf signal around $1 \mathrm{GHz}$ is injected. Relaxation oscillator operation of the RTD produces optical pulses as short as $33 \mathrm{ps}$ and with a modulation depth up to $18 \mathrm{~dB}$, for a range of amplitudes and frequencies of the injected signal. At this stage, development of the device concept appears to be a promising route toward a high-speed, low power, optoelectronic modulator, with operation extended to $1550 \mathrm{~nm}$ possible by using the InAlGaAs quaternary system.

J. M. L. Figueiredo acknowledges FCT-PRAXIS XXIPortugal for his Ph.D. grant.

${ }^{1}$ E. R. Brown, J. R. Soderstrom, C. D. Parker, L. J. Mahoney, K. M. Molvar, and T. C. McGill, Appl. Phys. Lett. 58, 2291 (1991).

${ }^{2}$ E. Ozbay, D. M. Bloom, D. H. Chow, and J. N. Schulman, IEEE Electron Device Lett. 14, 400 (1993).

${ }^{3}$ S. C. Kan, S. Wu, S. Sanders, G. Griffel, and A. Yariv, J. Appl. Phys. 69, 3384 (1991).

${ }^{4}$ A. F. Lann, E. Grumann, A. Gabai, J. E. Golub, and P. England, Appl. Phys. Lett. 62, 13 (1993).

${ }^{5}$ S. C. Kan, P. J. Harshman, K. Y. Lau, Y. Wang, and W. I. Wang, IEEE Photonics Technol. Lett. 8, 641 (1996).

${ }^{6}$ T. S. Moise, Y.-C. Kao, C. L. Goldsmith, C. L. Schow, and J. C. Campbell, IEEE Photonics Technol. Lett. 9, 803 (1997).

${ }^{7}$ E. R. Brown, C. D. Parker, S. Verghese, M. W. Geis, and J. F. Harvey, Appl. Phys. Lett. 70, 2787 (1997).

${ }^{8}$ S. Verghese, C. D. Parker, and E. R. Brown, Appl. Phys. Lett. 72, 2550 (1998).

${ }^{9}$ S. G. Mc Meekin, M. R. S. Taylor, and C. N. Ironside, IEE Proc.: Optoelectron. 143, 12 (1996).

${ }^{10}$ S. G. McMeekin, M. R. S. Taylor, B. Vögele, C. R. Stanley, and C. N. Ironside, Appl. Phys. Lett. 65, 1076 (1994). 